\title{
Implementation of a Postpartum Hemorrhage Safety Bundle at an Urban Safety-Net Hospital
}

\author{
Naima T. Joseph, MD, MPH ${ }^{1}$ Nikkia H. Worrell, $\mathrm{MD}^{2}$ Janice Collins, $\mathrm{CNM}^{3}$ \\ Melanie Schmidt, PharmD ${ }^{4}$ Grace Sobers, MSN, RN, WHNP-BC ${ }^{5}$ Kathlyn Hutchins, RN ${ }^{5}$ \\ E. Britton Chahine, MD ${ }^{6}$ Christine Faya, $\mathrm{MD}^{7} \quad$ Luanne Lewis, $\mathrm{BA}^{8} \quad$ Victoria L. Green, JD, MD, MBA ${ }^{6}$ \\ Penny Z. Castellano, MD ${ }^{6}$ Michael K. Lindsay, MD, MPH ${ }^{1}$
}

\footnotetext{
${ }^{1}$ Division of Maternal Fetal Medicine, Department of Gynecology and Obstetrics, Emory University School of Medicine, Atlanta, Georgia

${ }^{2}$ Department of Quality, Grady Health System, Atlanta, Georgia

3 Department of Obstetrics and Gynecology, Morehouse University School of Medicine, Atlanta, Georgia

${ }^{4}$ Department of Pharmacy, Grady Health System, Atlanta, Georgia

${ }^{5}$ Department of Nursing, Grady Health System, Atlanta, Georgia

${ }^{6}$ Division of General Obstetrics and Gynecology, Department of Gynecology and Obstetrics, Emory University School of Medicine, Atlanta, Georgia

7 Department of Anesthesia, Emory University School of Medicine, Atlanta, Georgia

${ }^{8}$ Health Information Management, Grady Health System, Atlanta, Georgia
}

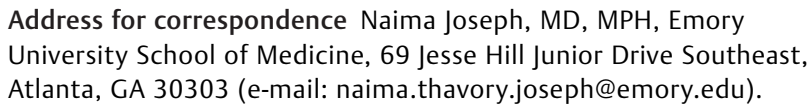

Atlanta, GA 30303 (e-mail: naima.thavory.joseph@emory.edu).

Am J Perinatol Rep 2020;10:e255-e261.

\begin{abstract}
Keywords

- postpartum hemorrhage

- obstetric hemorrhage

- safety-bundle

- safety-net hospital

Background Postpartum hemorrhage (PPH) is a leading cause of preventable maternal morbidity and mortality. Standardized response to obstetric hemorrhage is associated with significant improvement in maternal outcomes, yet implementation can be challenging.

Objective The primary objective is to describe the methodology for program implementation of the Alliance for Innovation on Maternal Health Safety Bundle on PPH at an urban safety-net hospital.

Methods Over an 18-month period, interventions geared toward (1) risk assessment and stratification, (2) hemorrhage identification and management, (3) team communication and simulation, and (4) debriefs and case review were implemented. Hemorrhage risk assessment stratification rates were tracked overtime as an early measure of bundle compliance.

Results Hemorrhage risk assessment stratification rates improved to $>90 \%$ during bundle implementation.

Conclusion Keys to implementation included multidisciplinary stakeholder commitment, stepwise and iterative approach, and parallel systems for monitoring and evaluation Implementation of a PPH safety bundle is feasible in a resource-constrained setting.
\end{abstract}

received

December 1, 2019 accepted after revision May 21, 2020
DOI https://doi.org/

10.1055/s-0040-1714713. ISSN 2157-6998.
Copyright $\odot 2020$ by Thieme Medical Publishers, Inc., 333 Seventh Avenue, New York, NY 10001, USA. Tel: +1(212) 760-0888.
License terms

(c) $(1) \ominus$ 
Postpartum hemorrhage (PPH) accounts for $11.4 \%$ of U.S. maternal deaths and is a leading cause of preventable pregnancy-related mortality. ${ }^{1-5}$ In 2017, the American College of Obstetricians and Gynecologists called for widespread hospital implementation of organized and systematic processes in PPH management. ${ }^{6}$ Effective July 1 , 2020, the Joint Commission will require hospitals to have evidence-based practice elements aimed toward preventing PPH-related maternal morbidity and mortality. ${ }^{7}$ The Alliance for Innovation on Maternal Health (AIM) Safety Bundle on $\mathrm{PPH}-$ adapted from the National Partnership for Maternal Safety's obstetrics (OB) Hemorrhage Bundle-is a tool that compiles evidence-based, peer-reviewed guidelines and contains 13 key practice elements which are organized into a form that aids implementation and consistency of practice. Use of an OB PPH Safety Bundle has been associated with improved PPH management and morbidity benefit. ${ }^{8,9}$

Grady Memorial Hospital is a large publicly supported hospital in Atlanta (970 beds, approximately 2,500 deliveries per year). Grady is staffed by faculty from two medical schools and serves as a safety net hospital, and a regional perinatal center, accepting high-risk patients from Georgia, Florida, and Tennessee. ${ }^{10}$ Examination of maternal outcomes at Grady found that PPH complicated $11 \%$ of deliveries and was responsible for $7.5 \%$ of preventable pregnancy-related deaths in a 40 -year period. ${ }^{11}$ Safety-net hospitals play a critical role in providing care to Medicaid, uninsured, undocumented, or otherwise vulnerable patients, yet their ability to provide sustained high-quality care is limited by resource availability and economic challenges. ${ }^{12,13}$ As a safety-net hospital providing care for high-risk parturients, we partnered with the Georgia Perinatal Quality Collaborative to implement the AIM PPH Safety Bundle.

In this article, we present key steps with insights that might assist groups contemplating implementation of similar initiatives in their hospitals. This was deemed exempt from institutional review board approval by both the Emory and Morehouse Schools of Medicine.

\section{Methods}

\section{Establishing an Obstetric Hemorrhage Task Force and Task Force Aims}

The labor and delivery unit is staffed by residents and faculty from two academic services, each with independent Chiefs of Service who report to the hospital's chief medical officer; nursing reports to the hospital's chief nursing officer. Prior to implementation, the three groups had been working independently to improve care processes on the unit. The chief medical officer requested that the chiefs of obstetrics and nursing leadership convene and assess PPH management in our hospital. A 10-member multidisciplinary task force was formed with representation from both obstetric services, maternal-fetal medicine, midwifery, obstetric anesthesia, labor and delivery nurses, postpartum nurses, nurse educators, pharmacy, health information management, and quality. The charge of the task force was to determine the incidence and severity of obstetric hemorrhage to review the differences in hemorrhage management, to develop a program mission, and to identify key interventions. Key deficiencies were identified through the process, which ultimately led to the following priority goals (1) systematic identification of all women at high risk for PPH; (2) standard and efficient recognition and management of $\mathrm{PPH}$; (3) ready access to hemorrhage management medications, instruments, and transfusion; (4) improvement in team-based performance; and (5) a process to conduct debriefs and detailed review of hemorrhage cases. A key driver diagram (-Fig. 1) was adapted from AIM and organized to include the program mission, aims, and key interventions for implementation. $^{14}$

\section{Improving Identification and Delivery Preparation}

The Association of Women's Health, Obstetric and Neonatal Nurses Postpartum Hemorrhage Risk Assessment Tool (PHRAT), uses an algorithm to combine the patient responses, clinical factors, and available chart data to systematically risk stratify all delivering women into low-, medium-, or high-risk categories (-Fig. 2). ${ }^{15,16}$ The initial risk assessment is performed by the patient's nurse at the time of delivery hospitalization admission and adjusted should the patient develop risk factors such as chorioamnionitis, preeclampsia, prolonged second stage, or difficult placental extraction.

The patient's risk classification is communicated three ways (Supplement) ${ }^{1}$ : In the electronic medical record (EMR), with a green, yellow, or red circle corresponding to her

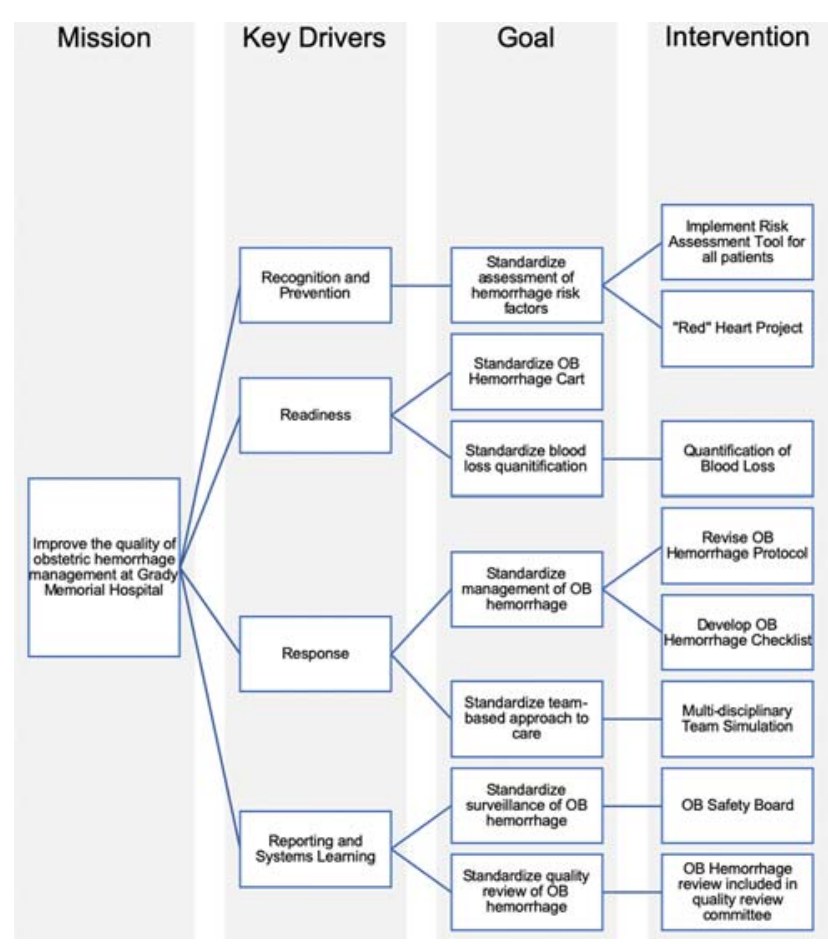

Fig. 1 Key driver diagram. Built on the "four Rs" from AIM, this depicts the program mission and organization includes the program's goal (based on the obstetric hemorrhage bundle from AIM), key drivers, and itemizes goals and intervention. Grady specific initiatives are listed under the Intervention section. AIM, Alliance for Innovation on Maternal Health. 


\begin{tabular}{|c|c|c|c|}
\hline \multicolumn{4}{|c|}{ POSTPARTUM HEMORRHAGE (PPH) RISK ASSESSMENT TABLE • 1.1} \\
\hline \multicolumn{4}{|c|}{$\begin{array}{l}\text { CLINICIAN GUIDELINES: } \\
\text { - Each box } 1 \text { represents ONE risk factor. Treat patients with } 2 \text { or more medium risk } \\
\text { factors as high risk. } \\
\text { - Prenatal risk assessment is beyond the scope of this document, however performing } \\
\text { a prenatal hemorrhage risk assessment and planning is highly recommended. Early } \\
\text { identification and management preparation for patients with special considerations } \\
\text { such as placental previa/accreta, bleeding disorder, or those who decline blood } \\
\text { products will assist in better outcomes. }\end{array}$} \\
\hline \multicolumn{4}{|c|}{ RISK CATEGORY: ADMISSION } \\
\hline & Low Risk & $\begin{array}{c}\text { Medium Risk } \\
\text { (2 or More Medium Risk Factors Advance Patient to High Risk Status) }\end{array}$ & High Risk \\
\hline & No previous uterine incision & Induction of labor (with oxytocin) or Cervical ripening & $\square$ Has 2 or More Medium Risk Factors \\
\hline & J Singleton pregnancy & a Multiple gestation & Active bleeding more than "bloody show" \\
\hline & \multirow[t]{2}{*}{$\square \leq 4$ Previous vaginal births } & $\sqsupset>4$ Previous vaginal births & $\square$ Suspected placenta accreta or percreta \\
\hline & & Prior cesarean birth or prior uterine incision & a Placenta previa, low lying placenta \\
\hline & No known bleeding disorder & $\square$ Large uterine fibroids & $\square$ Known coagulopathy \\
\hline & a No history of PPH & Jistory of one previous PPH & $\square$ History of more than one previous PPH \\
\hline & & $\begin{array}{l}\text { Family history in first degree relatives who experienced } \\
\text { PPH (known or unknown etiology with possible } \\
\text { coagulopathy) }\end{array}$ & a Hematocrit <30 AND other risk factors \\
\hline & & J Chorioamnionitis & a Platelets $<100,000 / \mathrm{mm} 3$ \\
\hline & & a Fetal demise & \\
\hline & & a Polyhydramnios & \\
\hline \multicolumn{4}{|c|}{$\begin{array}{l}\text { Anticipatory Interventions } \\
\text { Monitor patient for any change in risk factors at admission and implement anticipatory interventions as indicated. }\end{array}$} \\
\hline \multirow{3}{*}{$\begin{array}{l}\text { Blood Bank } \\
\text { Order: } \\
\text { Change } \\
\text { blood bank } \\
\text { orders as } \\
\text { needed if } \\
\text { risk catego- } \\
\text { ry changes }\end{array}$} & $\square$ Clot Only (Type and Hold) & Obtain Type and Screen & $\square$ Obtain Type and Cross (See Clinical Guidelines) \\
\hline & & $\begin{array}{l}\text { Notify appropriate personnel such as the Provider } \\
\text { (OB MD/CNM), Anesthesia, Blood Bank, Charge Nurse, } \\
\text { Clinical Nurse Specialist }\end{array}$ & $\begin{array}{l}\text { Notify appropriate personnel such as the Provider } \\
\text { (OB MD/CNM), Anesthesia, Blood Bank, Charge Nurse, } \\
\text { Clinical Nurse Specialist }\end{array}$ \\
\hline & & & $\begin{array}{l}\text { Consider delivering at a facility with the } \\
\text { appropriate level of care capable of managing } \\
\text { a high risk mother }\end{array}$ \\
\hline
\end{tabular}

02017 by the Association of Women's Health, Obstetric and Neonatal Nurses. All rights reserved. Requests for permission should be directed to permissions@awhonn.org. The Postpartum Hemorrhage (PPH) Risk Assessment Table is exemplary and does not include all possible patient complaints or conditions. The PPH Risk Assessment Table is designed to guide clinical decision-making but does not replace clinical judgment.

To access the full 3 page Risk Assessment Tool, users may visit www.AWHONN.org and enroll in the Postpartum Hemorrhage online education course.

Fig. 2 Postpartum Hemorrhage Risk Assessment Tool. Reprinted with permission from Association of Women's Health, Obstetric and Neonatal Nurses.

hemorrhage risk level ${ }^{2}$; color coded hearts placed on the door of every laboring woman ${ }^{3}$; verbally among providers during twice daily team huddles, which includes members from the obstetric, anesthesia, and nursing teams.

The patient's risk stratification guides delivery preparation and blood availability. The electronic record of all admitted patients is reviewed to confirm the patient's type and screen. Low-risk patients have a "clot to hold, blood" order placed, which instructs the nurse to draw blood and send the specimen to the blood bank, but no additional testing is performed. This allows for availability of crossmatched blood within 1 hour. Medium-risk patients have a "type and screen" order, which instructs the blood bank to perform $A B O$ group-Rh type-antibody screen and to have blood available within 15 minutes. High-risk patients (as well as antibody-positive patients) have an $\mathrm{ABO}$ group- $\mathrm{Rh}$ type-antibody screen-crossmatch order with immediate availability of blood. High-risk patients also have the obstetric hemorrhage cart placed outside their room at delivery. These orders are performed through the EMR via the "intrapartum and immediate postpartum admission" order set, which prompts the provider to risk stratification patient and appropriately select options to order additional IV placement, adjusted vital sign frequency monitoring, type and screen, crossmatch for blood, tranexamic acid, and postpartum uterotonic agents.

\section{Improving Recognition and Response}

\section{Quantitative Blood Loss}

The most common method of measuring blood loss during the third stage of labor is visual estimation of blood loss (EBL) by the birth attendant. Quantitative blood loss (QBL) has been proposed to be more accurate than EBL for the management of obstetric hemorrhage and has a higher sensitivity in diagnosing PPH. ${ }^{17-22}$ For vaginal deliveries, we used commercially available underbuttock drapes with a funneled, metered plastic bag to collect blood and amniotic fluid expelled during delivery. The volume of amniotic fluid was measured prior to placental delivery and subtracted from the total volume in the bag. Similarly, the volume of amniotic fluid was measured and subtracted from the total volume in the suction cannister during cesarean deliveries.

Weighed sponges also contributed to blood loss quantification. The sponges are weighed on the neonatal scales available in each room as follows: the sponges have a premeasured dry weight which is subtracted from the wet weight. That difference is converted in $1: 1 \mathrm{~g}$ to milliliters, 
which is added to the measured blood loss volume. If blood was collected on other materials (i.e., postpartum pads or sheets), the difference between the dry and wet weight would use to calculate QBL. The dry weight ratios for commonly saturated objects were kept on hemorrhage carts and laminated copies affixed to badges (Supplement).

\section{Obstetric Simulations and Performance Feedback}

All physician and nursing providers were required to complete online educational modules on hemorrhage management. Obstetric simulations reinforced team-approach to care. Examples of simulation exercises include QBL, using the $\mathrm{OB}$ hemorrhage cart, $\mathrm{OB}$ hemorrhage stage approach to intervention based on the $\mathrm{OB}$ hemorrhage protocol, and management of disseminated intravascular coagulation, and cardiopulmonary arrest. Over an 18-month time period, a total of fourteen 30-minute simulation exercises were conducted on PPH management, capturing approximately $85 \%$ of the labor and delivery workforce.

\section{Standardization of the Management of Postpartum Hemorrhage}

\section{Hemorrhage Protocol}

A revised hemorrhage protocol was implemented in close coordination with transfusion medicine, pharmacy, anesthesia, maternal fetal medicine, and obstetrics, following the California Maternal Quality Care Collaborative Obstetric Staged hemorrhage care guidelines checklist. ${ }^{23,24} \mathrm{We}$ worked with our health information system and transfusion medicine to streamline electronic activation of the Massive
Transfusion Protocol (MTP) by creating an order-sets that includes 6UPRBC-6U FFP, followed by 6UPRBC-6U FFP-1 pack platelets, alternating every 30 minutes until resolution, with cryoprecipitate ordered based on laboratory values.

A protocol focused on stage approach to interventions was developed (-Fig. 3). For example, Stage 0 managementwhich is defined as prevention-includes QBL, early administration of tranexamic acid or uterotonic agents if ongoing blood loss, and oxytocin administration using the Rule of 3 Algorithm after cesarean delivery or as a 10-unit bolus after vaginal delivery. ${ }^{6,25,26}$ The hemorrhage cart should be called for if there is concern for Stage 1 hemorrhage, as well as continued administration of uterotonic agents and fluid resuscitation; additional nursing or physician help should be mobilized. ${ }^{27}$ Stage 2 involves notification of an attending obstetrician, charge nurse, and $\mathrm{OB}$ anesthesia; possible mobilization to the operating room if not already there; and blood transfusion while the back up team is mobilized, MTP is activated, and the patient definitively brought to the operating room for all Stage 3 hemorrhages. Additionally, a time-keeper and recorder is identified, and the OB Narrator used to document interventions. These patients are admitted either to obstetric intermediate care unit on labor and delivery or the intensive care unit following resolution.

\section{Defining Team Members, Roles, and Responsibilities}

We defined the hemorrhage response team to include the delivering attending physician, the resident physician, the patient's primary nurse, the charge nurse, and anesthesia. For Stage 3 hemorrhage, we created a tiered back-up system which included a second obstetric attending physician and

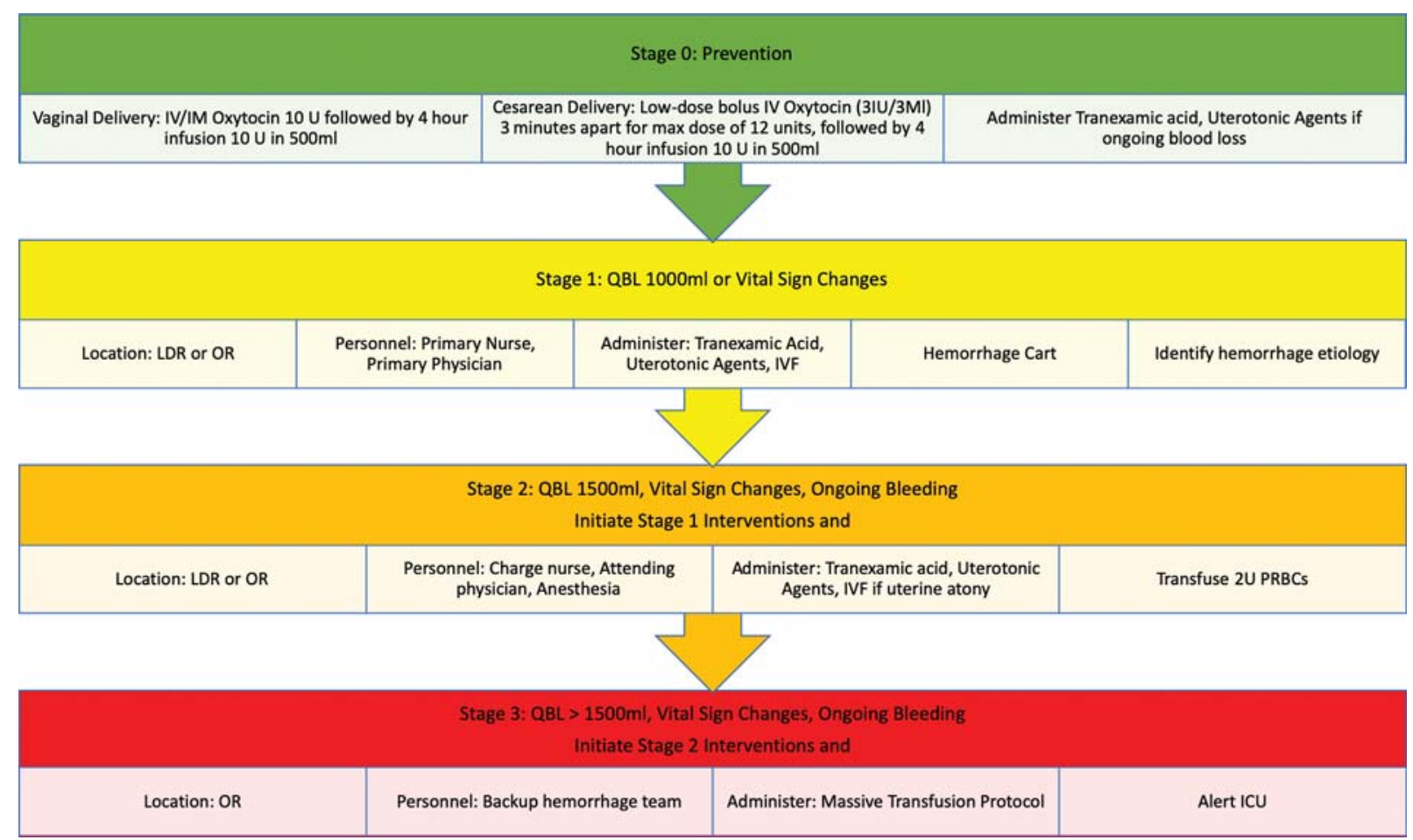

Fig. 3 Obstetrics Hemorrhage Protocol. Pictorial depiction of the protocol with a staged approach to intervention. ICU, intensive care unit; IVF, intravenous fluid such as plasma-lyte, lacted ringer, or normal saline; LDR, labor and delivery room; OR, operating room. 
additional nurse. The on-call maternal fetal medicine attending, advanced gynecologic surgeon, urology, and trauma services were involved when needed.

\section{Obstetric Hemorrhage Cart}

As a key component of our initiative, OB hemorrhage carts were designed with input from pharmacy, obstetric, and nursing providers. The purpose of the hemorrhage cart was to have readily available checklists, uterotonic medications, and other supplies required to manage refractory hemorrhage. A total of five hemorrhage carts were developed: three of which are stationed on labor and delivery, one in the operating room suite, and one in the postpartum unit. The hemorrhage protocol is affixed to the top of the cart, as well as the names, dose, and route of administration for uterotonic medications and tranexamic acid ${ }^{28}$; all contain uterotonic medications, surgical instruments, kits for laboratory draws, and materials for fluid administration (Supplement).

\section{Postpartum Hemorrhage Order-Set}

The OB narrator (Supplement) is a tool to document hemorrhage management and a checklist, ensuring appropriate management of hemorrhage in real time. It can therefore be used for team debriefs, case reviews, and process improvement. The paper form is under pilot, with the ultimate goal is an electronic version that can also function as an order set, which would include MTP activation.

\section{Team Debriefs}

We created a formal process to conduct debriefs and detailed review of PPH cases. Team Debriefs occur after management of any patient with $\mathrm{PPH}$, immediately following patient stabilization. Stage 3 hemorrhage review involves chart investigation and presentation at Mortality and Morbidity conference, which provides for department wide review and process improvement. These cases are also brought to the Perinatal Quality Committee, allowing for multidisciplinary input. The purpose of these reviews is to provide a protected forum for process evaluation, root cause analysis, and systems improvement.

\section{Monitoring and Evaluation}

For each intervention detailed, the task force developed a plan for stepwise implementation and monitored integration. Safety bundle implementation was iterative, requiring multiple Plan-Do-Study-Act (PDSA) cycles, continuous nurse training, and completion rate reports. Interventions were introduced during twice daily team huddles and formal didactic conferences for nurses, midwives, and physicians. Target metrics were developed, collected, and displayed on a team progress board on the unit. Consultants were hired to perform external quarterly review of the program.

The most reliable metric we developed was PHRAT completion rate, which was generated using the EMR. We collected process metrics (case reviews rates, proportion of providers/nurses educated, PHRAT, and QBL completion rates) and outcome metrics (PPH rates) initially were collected and available data kept on a team progress board on the unit. When targets were not achieved, case review involving chart investigation and discussion with the delivery team was conducted. Individual and group feedback was provided by physician and nursing leadership, and multidisciplinary buy-in and was critical to improving nonadherence and achieving targets. For example, PHRAT completion rates (-Fig. 4)-measured as the proportion of delivering women with a risk assessment completed prior to delivery-improved once we addressed different workflows for patients transferred to labor and delivery from other hospital units, identified a nurse educator to lead nursing teaching and utilization, and provided continuous verbal and visual feedback on performance. Protocols were changed often, sometimes daily, to improve use.

As we developed EMR capability for surveillance, we developed a rudimentary monitoring and evaluation system involving an OB safety board. The goal was to capture rates of Stage 3 hemorrhage, ICU admission, blood transfusion greater than 4 units; the MRN for any patient with these outcomes was added to a poster board. The "safety board" was placed in a Health Insurance Portability and Accountability Act-compliant office, accessible only to physicians, midwives, and nurses on the unit and the location where team huddles occur. EMR reports involve using International Classification of Diseases-10 and procedure codes to track outcomes, remains an ongoing area of improvement. ${ }^{29}$

\section{Discussion}

This paper describes key components for the implementation of a standardized protocol for improved surveillance and management of obstetric hemorrhage (-Table 1). The process was iterative process, requiring multidisciplinary, high-level, hospital administrative engagement, as well as physician and nursing co-champions for clinical integration. Critical components toward successful integration include culture change to one geared toward patient safety, weekly meetings during which implementation processes were reviewed, and nursing involvement who provided behavioral motivation, educational support, and advocated for enhanced communication between all members of the team. Continuous education, feedback, and intensive audit is critical.

Regarding actual implementation, the order in which to implement the initiatives is critically important. After the task force was convened, the first initiative was to create the obstetric narrator to standardize hemorrhage management. It became clear that this would be insufficient to standardize and improve our management and instead we focused our attention on effecting a proactive approach to hemorrhage management rather than reactive.

Based on our experience with this multicomponent implementation, the following sequence for implementation is recommended:

- Standardize a hemorrhage protocol

- Define a hemorrhage response team and ensure ongoing education on hemorrhage management for all providers on the unit 


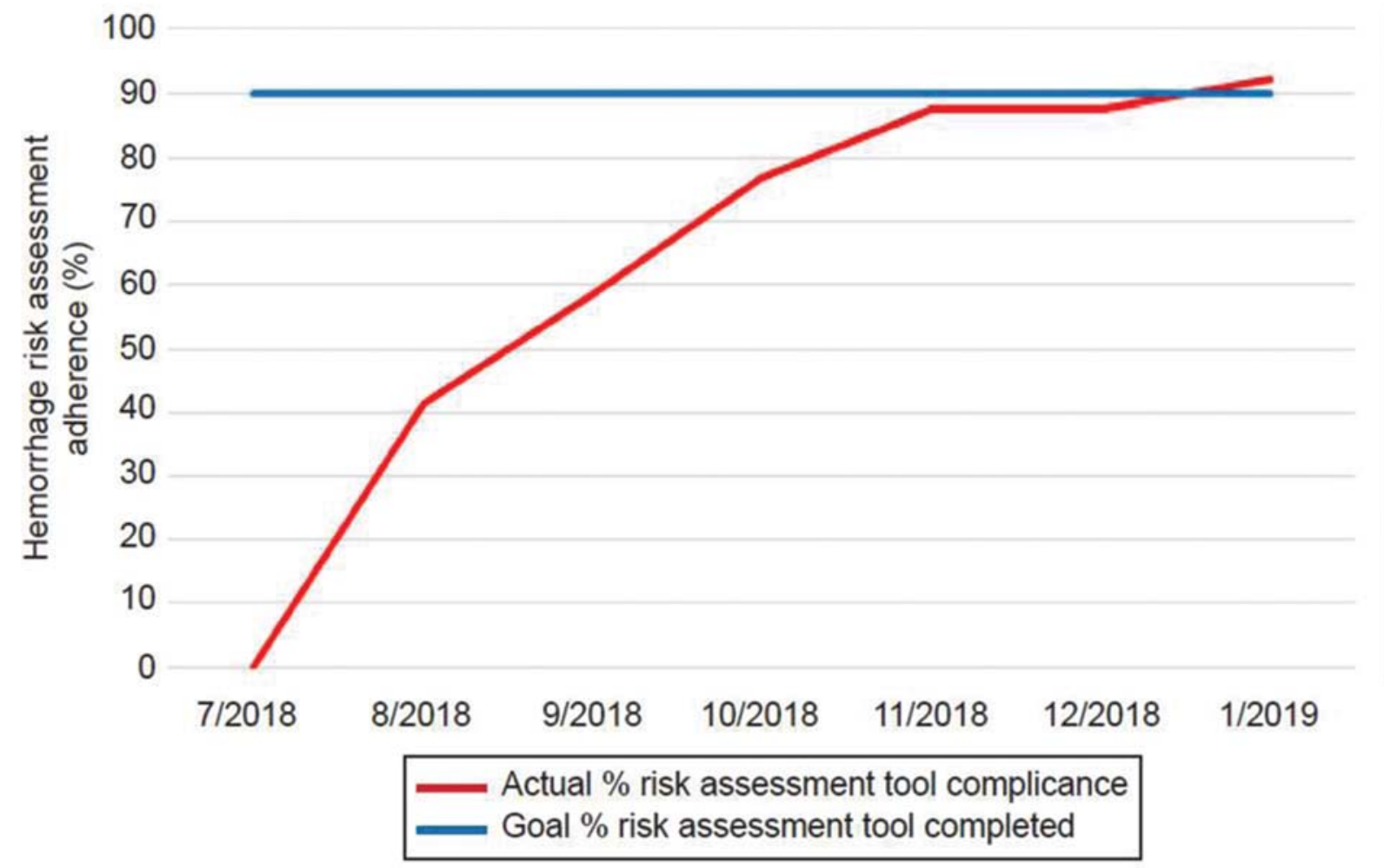

Fig. 4 Risk assessment tool compliance rates. This is an example of how compliance rates improve and the need for iterative approach to integration. The rates were reported weekly and then monthly, and posted on the team communication board on labor and delivery. In-depth reviews, including review of the patient's medical record, admission note, and discussion with the admitting nurse, was performed for all patients missing assessments.

Table 1 Prerequisites to effective implementation of postpartum hemorrhage bundle

Clearly define the burden of hemorrhage and align proposed change to institutional goals

Develop a strategy for open communication and involve key stakeholders

Provide effective training and iterative implementation of initiatives

Develop infrastructure support

Monitor and evaluate process measures and health outcomes

- Routinize use of Postpartum Hemorrhage Risk Assessment Tool and establish an easy method to communicate risk status to team members (i.e., Red Hearts)

- Employ QBL for all deliveries

- Develop and utilize hemorrhage carts

- Streamline protocol adherence with an obstetric narrator

Systems for case review and monitoring and evaluation (i.e., simulation, chart review and debrief, OB Safety board) should also be implemented in parallel.

A major limitation of the process is that a system for monitoring and evaluation was not established prior to the start of the safety bundle implementation. Further, we did not formalize a process to assess unintended consequences from the program. For example, the risk assessment tool stratifies $49 \%$ of our patients as high risk for PPH, yet only $7-$ $9 \%$ of deliveries are complicated by PPH. It is unclear how the burden of excess preparation has affected transfusion services or services at our institutions. From the outset, it is important to develop process and outcome metrics to track progress; not only for process improvement, but also for positive reinforcement.

National organizations have called for the implementation of an obstetric hemorrhage bundle in all maternity hospitals, which is now a standard for accreditation required by the Joint Commission. ${ }^{7}$ It is our goal that this paper may provide anticipatory guidance for other organizations beginning to implement an obstetric hemorrhage bundle, and that others may learn from our experience.

Conflict of Interest

None declared.

\section{References}

1 Creanga AA, Syverson C, Seed K, Callaghan WM. Pregnancyrelated mortality in the United States, 2011-2013. Obstet Gynecol 2017;130(02):366-373

2 Bateman BT, Berman MF, Riley LE, Leffert LR. The epidemiology of postpartum hemorrhage in a large, nationwide sample of deliveries. Anesth Analg 2010;110(05):1368-1373

3 Berg CJ, Harper MA, Atkinson SM, et al. Preventability of pregnancy-related deaths: results of a state-wide review. Obstet Gynecol 2005;106(06):1228-1234 
4 Della Torre M, Kilpatrick SJ, Hibbard JU, et al. Assessing preventability for obstetric hemorrhage. Am J Perinatol 2011;28(10):753-760

5 Berg CJ, Callaghan WM, Syverson C, Henderson Z. Pregnancyrelated mortality in the United States, 1998 to 2005. Obstet Gynecol 2010;116(06):1302-1309

6 Shields LE, Goffman D, Caughey AB. Practice bulletin no. 183: postpartum hemorrhage. Obstet Gynecol 2017;130(04):168-186

7 The Joint Commission. Provision of care, treatment, and services standards for maternal safety. Require Ration Ref 2019;24:1-6

8 Main EK, Goffman D, Scavone BM, et al; National Parternship for Maternal Safety; Council for Patient Safety in Women's Health Care. National Partnership for Maternal Safety: consensus bundle on obstetric hemorrhage. Anesth Analg 2015;121(01):142-148

9 Berg C, Danel I, Atrash H, Zane S, Linda B. Strategies to reduce pregnancy-related deaths: from identification and review to action. Atlanta; 2001. Available at: www.cdc.gov/nccdphp/drh/. Accessed 2001

10 Becker's Hospital Review. 25 largest public hospitals in America. Available at: https://www.beckershospitalreview.com/lists/25largest-public-hospitals-in-america-2015.html. Accessed 2015

11 Manley C, Zahedi-Spung L, Ellis J, Brown J, Lindsay MK. Abstract 248: Leading causes of maternal mortality at an inner-city Hospital, 1949-2017. Am J Obstet Gynecol 2019;220(01):S179-S180

12 Bachrach D, Braslow L, Karl A, Bruce S. Toward a high performance health care system for vulnerable populations: funding for safetynet hospitals. Prepared for the Commonwealth Fund Commission on a High Performance Health System. Available at: www.commonwealthfund.org. Accessed 2012

13 Werner RM, Goldman LE, Dudley RA. Comparison of change in quality of care between safety-net and non-safety-net hospitals. JAMA 2008;299(18):2180-2187

14 Council on Patient Safety in Women's Health Care. Obsetric hemorrhage safety bundle. Available at: https://safehealthcareforeverywoman.org/wp-content/uploads/2017/11/Obstetric-Hemorrhage-Bundle.pdf. Accessed 2015

15 Kleinman A, Deeb J, Cassidy M, Richter S. The Hemorrhage Risk Score. J Obstet Gynecol Neonatal Nurs 2017;46(Supplement 3S): S25. Doi: 10.1016/j.jogn.2017.04.046

16 The AWHONN Postpartum Hemorrhage Project. Postpartum hemorrhage ( $\mathrm{PPH})$ risk assessment table. Available at: https:// mygnosis.com/Content/Chunks/3504/assets/pdfs/PPH_Risk_Assessment_Table-7-17-15.pdf. Accessed 2015

17 Le NK, Briceno M, Brown A, Leavitt K, Louis J. Abstract: efficacy of quantitative blood loss compared to estimated blood loss for predicting severe obstetric hemorrhage. Obstet Gynecol 2018; 131(05):2018

18 Patel A, Goudar SS, Geller SE, et al. Drape estimation vs. visual assessment for estimating postpartum hemorrhage. Int J Gynaecol Obstet 2006;93(03):220-224

19 Diaz V, Abalos E, Carroli G. Methods for blood loss estimation after vaginal birth. Cochrane Database Syst Rev 2018;9(09):CD010980

20 Duzyj CM, Johnson AR, Mahoney K. Abstract 162: a comparison of estimated blood loss and quantitative blood loss as predictors of peripartum hemoglobin change. Am J Obstet Gynecol 2018;218 (01):S112

21 Gabel KT, Weeber TA. Measuring and communicating blood loss during obstetric hemorrhage. J Obstet Gynecol Neonatal Nurs 2012;41(04):551-558

22 Smith CM, Borders AE, King TL. ACOG committee opinion no. 794. Quantitative blood loss in obstetric hemorrhage. Obstet Gynecol 2019;134(794):150-156

23 Bingham D, Melsop K, Main E. CMQCC. The obstetric hemorrhage toolkit hospital level implementation guide. Available at: https:// www.cmqcc.org/resources-tool-kits/toolkits/ob-hemorrhagetoolkit. Accessed July 2010

24 Burtelow M, Riley E, Druzin M, Fontaine M, Viele M, Goodnough LT. How we treat: management of life-threatening primary postpartum hemorrhage with a standardized massive transfusion protocol. Transfusion 2007;47(09):1564-1572

25 Begley CM, Gyte GM, Devane D, McGuire W, Weeks A, Biesty LM. Active versus expectant management for women in the third stage of labour. (Review)Cochrane Database Syst Rev 2019;2(02): CD007412

26 Kovacheva VP, Soens MA, Tsen LC. A randomized, double-blinded trial of a "rule of threes" algorithm versus continuous infusion of oxytocin during elective cesarean delivery. Anesthesiology 2015; 123(01):92-100

27 Shakur H, Roberts I, Fawole B, Collaborators WT; WOMAN Trial Collaborators. Effect of early tranexamic acid administration on mortality, hysterectomy, and other morbidities in women with post-partum haemorrhage (WOMAN): an international, randomised, double-blind, placebo-controlled trial. Lancet 2017;389 (10084):2105-2116

28 Harrison TK, Manser T, Howard SK, Gaba DM. Use of cognitive aids in a simulated anesthetic crisis. Anesth Analg 2006;103(03):551-556

29 Baier AW, Snyder DJ, Leahy IC, Patak LS, Brustowicz RM. A shared opportunity for improving electronic medical record data. Anesth Analg 2017;125(03):952-957 